\title{
Ordinary Muon Capture for Double Beta Decay and Anti-Neutrino Nuclear Responses
}

\author{
Izyan Hazwani Hashim ${ }^{1,2,3 *}$ and Hiroyasu Ejiri ${ }^{3}$ \\ ${ }^{1}$ Department of Physics, Faculty of Science, Universiti Teknologi Malaysia, Johor Bahru, Malaysia, ${ }^{2}$ National Centre for Particle \\ Physics, Universiti Malaya, Kuala Lumpur, Malaysia, ${ }^{3}$ Research Center for Nuclear Physics, Osaka University, Osaka, Japan
}

This is a brief review on ordinary muon capture (OMC) experiments at Research Center for Nuclear Physics (RCNP) Osaka University relevant for the study of double beta decays (DBDs) and astro anti-neutrinos (neutrino) nuclear responses. OMC usually leaves the nucleus in highly excited unbound state. $\mathrm{OMC}$ is a charge exchange reaction via the charged weak boson as given by $\left(\mu, v_{\mu}\right)$ reactions with $\mu$ and $v_{\mu}$ being the muon and muon neutrino. Subjects discussed include 1) unique features of $\mathrm{OMC}$ for studying DBDs and astro anti-neutrino (neutrino) nuclear responses, 2) experiments of OMCs on ${ }^{100} \mathrm{Mo}$ and ${ }^{\text {nat }}$ Mo to study neutrino nuclear responses for DBDs and astro anti-neutrinos, 3) impact of

OPEN ACCESS

Edited by:

Theocharis S. Kosmas, University of loannina, Greece

Reviewed by: Dieter Frekers,

University Muenster, Germany Frank Franz Deppisch, University College London, United Kingdom

${ }^{*}$ Correspondence: Izyan Hazwani Hashim izyan@utm.my

Specialty section: This article was submitted to High-Energy and Astroparticle Physics, a section of the journa Frontiers in Astronomy and Space Sciences

Received: 10 February 2021 Accepted: 05 May 2021 Published: 28 May 2021

Citation: Hashim IH and Ejiri H (2021) Ordinary Muon Capture for Double Beta Decay and Anti-Neutrino Nuclear Responses. Front. Astron. Space Sci. 8:666383. doi: 10.3389/fspas.2021.666383 the OMC results on neutrino nuclear responses for DBDs and astro anti-neutrinos. Remarks and perspectives on OMC experiments for neutrino nuclear responses are briefly described.

Keywords: ordinary muon capture, muon charge exchange reaction, neutrino nuclear response, double beta decay, supernova neutrino, nuclear matrix element, neutrino mass

\section{INTRODUCTION}

Double beta decays (DBDs) and astro (solar and supernova) neutrinos and anti-neutrinos are of current interest. Neutrino-less DBDs are used to explore the fundamental neutrino properties such as the Majorana nature, the absolute mass scale, the mass hierarchy and the $\mathrm{CP}$ phases beyond the standard electroweak model. The zero-neutrino $(0 v)$ DBD rate is given by $R^{0 v}=G^{0 v} \times\left|M^{0 v}\right|^{2} \times\left|m^{e f f}\right|^{2}$, where $G^{0 v}$ is the phase space volume, $m^{\text {eff }}$ is the effective neutrino mass and $M^{0 v}$ is the zero-neutrino DBD nuclear matrix element, whose squared value is the nuclear response, i.e: $B^{0 v}=\left|M^{0 v}\right|^{2}$. Here we consider the ground state $\left(0^{+}\right)$to ground state $\left(0^{+}\right)$transition ${ }_{Z}^{A} \mathrm{X} \rightarrow{ }_{Z+2}^{A} \mathrm{X}$. The DBD nuclear matrix element (NME) $M^{0 v}$ is given by the coherent sum of the individual matrix elements $M_{i}^{0 v}$ which connect the initial and final ground states via the $i$ th intermediate nucleus ${ }_{Z+1}^{A} \mathrm{X}_{i}$, where these extend up to about $100 \mathrm{MeV}$.

The supernova nucleo-synthesis rates induced by neutrinos and anti-neutrinos are proportional to the respective nuclear responses

$$
B^{\nu, \bar{\nu}}=\frac{1}{2 J_{A}+1}\left|M^{\nu, \bar{\nu}}\right|^{2}
$$

where $M^{\gamma, \bar{v}}$ are the NMEs, and $\left(2 J_{A}+1\right)$ is the spin factor for the initial nucleus. Note that the NMEs $M^{\gamma, \bar{\nu}}$ are the coherent sums of individual NMEs $M_{i}^{\nu, \bar{\nu}}$

$$
M^{\gamma, \bar{\nu}}=\sum_{i} M_{i}^{\nu, \bar{\nu}}
$$


for each intermediate state $(i)$ and in the respective isospin directions $\tau^{-}$for $v$ or $\tau^{+}$for $\bar{v}$. Note that the $v$ and $\bar{v}$ NMEs for the $i$ th intermediate state are given by the $\tau^{-}$and $\tau^{+}$NMEs of $M_{i}^{-}$and $\mathrm{Mi}_{i}^{+}$, respectively. Accordingly one needs the $B^{0 v}$ response to derive the effective neutrino mass $m^{\text {eff }}$ from the neutrino-less DBD rate and the astro neutrino (anti-neutrino) response $B^{V}\left(B^{\bar{\gamma}}\right)$ to derive the synthesis rate from the neutrino (anti-neutrino) flux. The neutrino nuclear responses are evaluated theoretically. However, the theoretical calculations for the neutrino nuclear responses for $\mathrm{DBD}$ and astro neutrino (anti-neutrino) depend much on the nuclear models and the nuclear parameters used. The neutrino nuclear responses and DBDs are extensively discussed in review articles and references therein (Ejiri, 2000; Vergados et al., 2012).

Experimental studies for the neutrino nuclear responses are interesting to provide the nuclear parameters to be used for theoretical calculations. Charge exchange reactions (CERs) using light ions have been used to study the neutrino nuclear responses. In particular, high energy-resolution $\left({ }^{3} \mathrm{He}, t\right)$ CERs have been used to study the neutrino nuclear responses for nuclei of DBD and astro neutrino interests in the broad energy and momentum regions. The responses studied by these light-ion CERs are the $\tau^{-}$-side ones, and the NMEs $M_{i}^{-}$are derived from CERs. On the other hand, the $\tau^{+}$-side responses and the NMEs $M_{i}^{+}$are not well studied since the $\left(t{ }^{3} \mathrm{He}\right)$ CERs to be used for studying the $\tau^{+}$-side responses require the radioactive $t$ beam, and thus high precision measurements are difficult. Medium energyresolution measurements are made by using $\left(d,{ }^{2} \mathrm{He}\right)$ reactions. Neutrino nuclear responses studied by these light-ion CERs are discussed in the recent review articles and references therein (Ejiri, 2000; Vergados et al., 2012; Frekers and Alanssari, 2018; Ejiri et al., 2019; Ejiri, 2020).

Recently ordinary muon capture reactions (OMC), where a negative muon in an atomic orbit is captured into the nucleus has been shown useful for studying the $\tau^{+}$-side responses, and the $\mathrm{M}_{i}^{+}$ NMEs at Research Center for Nuclear Physics (RCNP) Osaka University (Hashim, 2015; Hashim et al., 2018; Hashim and Ejiri, 2019). The present report is a brief mini-review on the OMC studies for the DBD and astro anti-neutrinos responses and related subjects on nuclear isotope productions at RCNP.

\section{UNIQUE FEATURES OF OMCS FOR NEUTRINO NUCLEAR RESPONSES}

In OMC some of the unique feature are as follows:

(1) OMC can be used to study the $\tau^{+}$-responses of proton $(\mathrm{p}) \rightarrow \operatorname{neutron}(\mathrm{n})$ in the nucleus, and the NME $M_{i}^{+}$for DBD and astro anti-neutrino.

(2) OMC transfers energy between 0-50 MeV and a momentum up to $100 \mathrm{MeV} / \mathrm{c}$ to the nucleus that are similar to those involved in neutrino-less DBD and supernova antineutrino.

(3) A negative muon is finally captured into the nucleus via the weak interaction. The capture probability in the medium and heavy nuclei is around $95 \%$ after a mean lifetime of about $100 \mathrm{~ns}$ in the atomic orbit. Low momentum (a few $10 \mathrm{MeV} / \mathrm{c}$ ) beam muons at intensities of $10^{3}-10^{4}$ muons/s are used for OMC studies.

(4) The OMC on ${ }_{Z}^{A} \mathrm{X}$, where $A$ and $Z$ are the mass and the atomic numbers, excites the nucleus ${ }_{Z-1}^{A} \mathrm{X}$ up to around $50 \mathrm{MeV}$, which decays by emitting a number $(x)$ of mostly neutrons and gamma rays to the ground state of the residual nucleus ${ }_{Z-1}^{A-x} \mathrm{X}$. The number of neutrons reflects the excitation energy. Accordingly, the relative strength as a function of the excitation energy is evaluated by measuring the number $(x)$ of the neutrons, i.e. the mass distribution $A-x$ of the residual nuclei.

(5) Absolute OMC rate is obtained from the measured lifetime of the trapped muons, and thus the neutrino nuclear responses are derived from the OMC rate.

\section{NEUTRINO NUCLEAR RESPONSES FOR MO ISOTOPES BY OMC}

The intense $400 \mathrm{MeV}$ proton beam with an intensity around $1 \mu \mathrm{A}$ from the RCNP cyclotron is used to produce pions. The momentum $(p \approx 30-50 \mathrm{MeV} / \mathrm{c}$ ) negative muons produced by the $\pi$-decays are guided by the MuSIC beamline to the target port. They are stopped in the target and are trapped in the inner orbit of the atom. Then, after around $100 \mathrm{~ns}$, the muon is mainly (around 95\%) captured into the nucleus via the OMC. The $\mu$-beam spot and the muon beam intensity at the target port are around $6 \mathrm{~cm} \times 6 \mathrm{~cm}$ and $5 \times 10^{3}$ per second (Hino et al., 2014). The $\mu$ capture rate is derived by measuring the electrons' time signal from the weak decay of the $\mu$ trapped in the atomic orbit by plastic scintillation detectors. The residual isotopes are identified by measuring characteristic prompt $\gamma$-rays of the residual nucleus by HPGe detectors online and delayed $\gamma$-rays from $\beta$-decays of residual isotopes by HPGe detectors offline.

Recently OMCs were studied on ${ }^{\text {nat }} \mathrm{Mo}$ and ${ }^{100} \mathrm{Mo}$, which are interesting for DBD and astro neutrino studies (Ejiri et al., 2019). The OMC on ${ }_{42}^{A}$ Mo isotopes with the mass-number $A=92-100$ produces ${ }_{41}^{A} \mathrm{Nb}$ with the excitation energy $E \approx 0-50 \mathrm{MeV}$. The excited $\mathrm{Nb}$ isotope decays mostly by emitting a number $10(x)$ of neutrons until the final state gets particle-bound. Then it decays by emitting prompt $\gamma$ rays to the ground state of ${ }^{A-x} \mathrm{Nb}$, which is followed by beta decay with comparatively long half-lives.

The OMC strength distribution as a function of the excitation energy $E$ is derived from the mass-number $(A-x)$ distribution $(x$ distribution) through the particle cascade model (Hashim et al., 2018; Hashim \& Ejiri, 2019; Hashim et al., 2020). The excited states in $\mathrm{Nb}$ isotopes decay by emitting mostly neutrons since the Coulomb barrier much suppresses proton emission. Thus, the neutron cascade emission model (NEM) (Hashim et al., 2017; Hashim et al., 2018) was developed to deduce the excitation energy in the initial isotope of ${ }^{A} \mathrm{Nb}$ from the number $10(x)$ of the emitted neutrons, i.e., the mass-number $A-x$ of the residual isotope ${ }^{A-x} \mathrm{Nb}$ after the $x$ neutron emission. The neutron energy spectrum for the first neutron consists of the preequilibrium (PEQ) and equilibrium (EQ) stages (Ejiri and de Voight, 1989). It is given as 


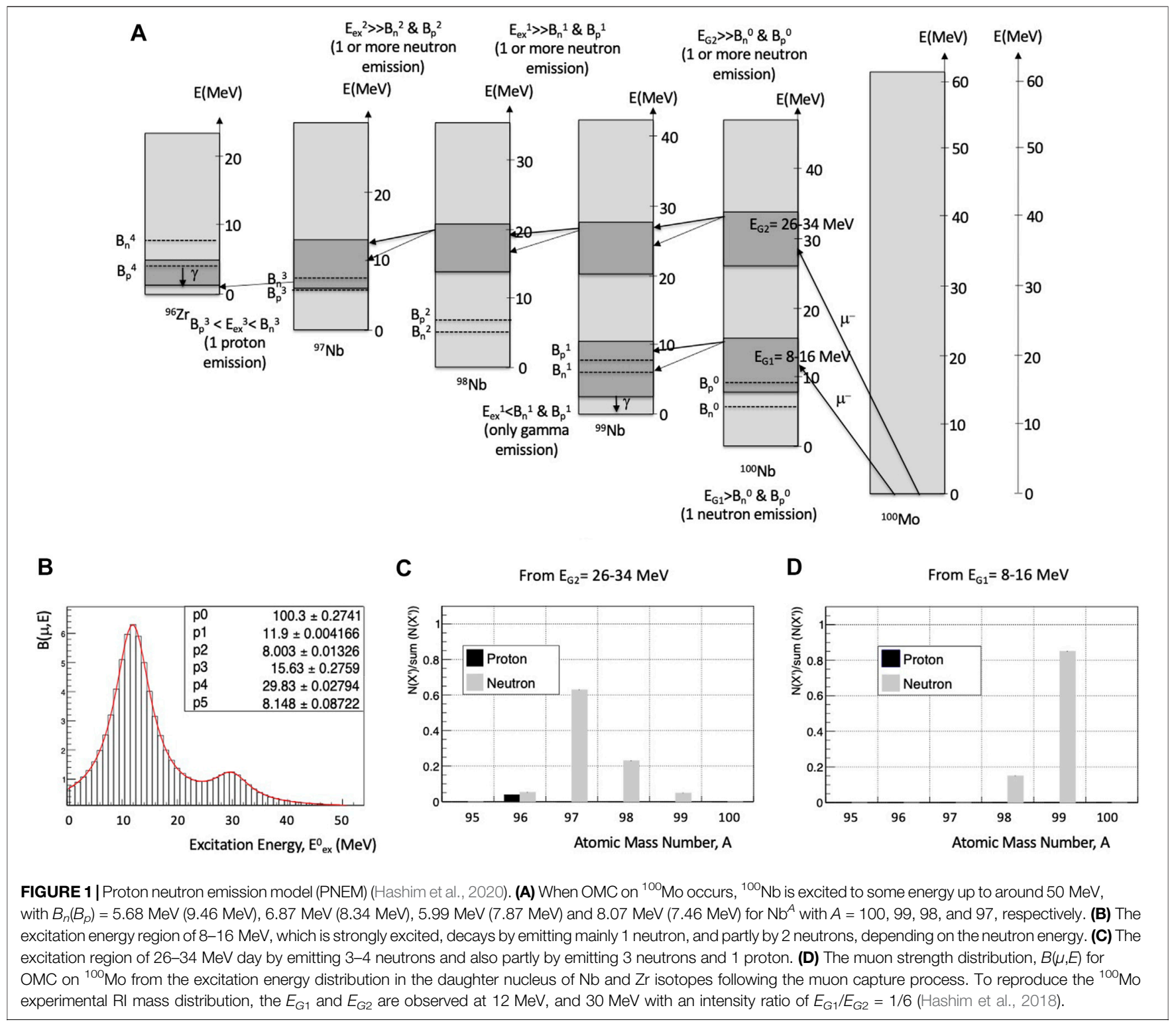

$$
S\left(E_{n 1}\right)=k\left[E_{n 1} \exp \left(-\frac{E_{n 1}}{T_{E Q}(E)}\right)+p E_{n 1} \exp \left(-\frac{E_{n 1}}{T_{P E Q}(E)}\right)\right]
$$

where $T_{\mathrm{EQ}}(E)$ and $T_{\mathrm{PEQ}}(E)$ are the $\mathrm{EQ}$ and $\mathrm{PEQ}$ nuclear temperatures, respectively. $T_{\mathrm{EQ}}(E)$ is given as a function of excitation energy $E$ (Ejiri and de Voight, 1989). The ratio of $T_{\mathrm{PEQ}}(E) / T_{\mathrm{EQ}}(E)=3$ for the medium excitation $10<E<40 \mathrm{MeV}$. After one neutron emission, the emission takes place only via the EQ stage. The OMC strength distribution for ${ }^{100} \mathrm{Nb}$ is derived from the observed mass-number $(A-x)$ distribution for the OMC on ${ }^{100} \mathrm{Mo}$.

The NEM analysis on the observed mass-number distribution of ${ }^{100-x} \mathrm{Nb}$ shows preferential excitation (muon giant resonance, $\mu$-GR) at $10-14 \mathrm{MeV}$ region and a broad bump at the higher excitation region of $25-40 \mathrm{MeV}$. The NEM analyses on other medium-heavy nuclei by (Ibrahim, 2018) using experimental data from (Measday et al., 2007b; Measday et al., 2007a) show similar features of the preferential excitation of the $10-15 \mathrm{MeV}$. This preferential excitation reflects the large branch of the one neutron $(x=1)$ emission. The NME analyses on the mass-number distributions for OMCs on light nuclei show a preferential excitation around $4-8 \mathrm{MeV}$ region by (Muslim, 2018) using experimental data from (Evans, 1973; Measday et al., 2007c). This observation is consistent with the calculation in (Kortelainen and Suhonen, 2004).

The strength distribution is fitted by the sum of the $\mu$-GR strengths of $B_{1}\left(\mu, E_{1}\right)$ and $B_{2}\left(\mu, E_{2}\right)$ given by

$$
B(\mu, E)=\sum_{i=1,2} B_{i}\left(\mu, E_{i}\right), B_{i}\left(\mu, E_{i}\right)=\frac{B_{i}(\mu)}{\left(E-E_{\mathrm{Gi}}\right)^{2}+\left(\Gamma_{i} / 2\right)^{2}}
$$

where $E_{\mathrm{Gi}}$ and $\Gamma_{i}$ with $i=1,2$ are the resonance energy and the width for the $i$ th GR, and the constant $B_{i}(\mu)$ is given by $\sigma_{i} \Gamma_{i} /(2 \pi)$ with $\sigma_{i}$ being the total strength integrated over the excitation 


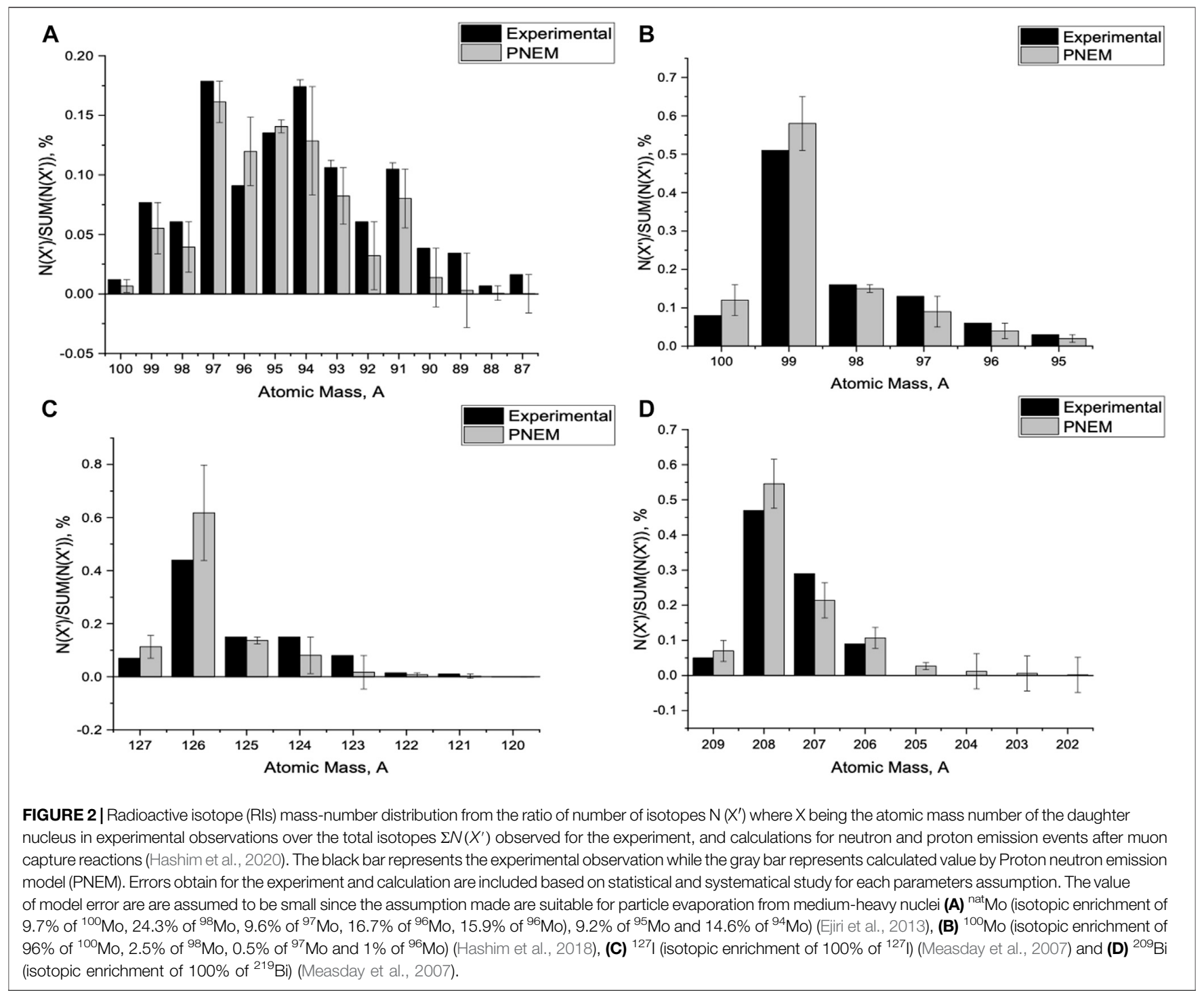

energy. The obtained GR energies are $12 \mathrm{MeV}$ for the first GR and $32.5 \mathrm{MeV}$ for the second GR, and the widths for both GRs are $8 \mathrm{MeV}$, as shown in Figure 1B. Here we note the GR2 is not entirely clear beyond the statistical error and maybe a broad bump. The $\mu$-GR energy peaks at around $12 \mathrm{MeV}$, which is lower than the giant dipole resonance (GDR) energy of $14 \mathrm{MeV}$ deduced from a photoninduced reaction (Ejiri et al., 2011). It also features a broader width of about $8 \mathrm{MeV}$ compared to the $5 \mathrm{MeV}$ width of the latter. This is, of course due to the additional multipole components of higher spin states excited by $\tau^{+}$, i. e.,: (n, $p$ )-type, muon capture.

Recently a proton-neutron emission model (PNEM) has been developed by considering the probability of proton emission as well (Hashim et al., 2020). The proton energy spectrum is given by $S\left(E_{p}\right)=m \times \exp (-n \times E p)$, where $m$ and $n$ are the density parameter constant with the value of $0.832 \mathrm{MeV}$ and $0.163 \mathrm{MeV}^{-1}$. Here, the proton is assumed to be emitted in the case of $B_{p} \leq E \leq B_{n}$ with $B_{P}, E$ and $B_{n}$ being the proton binding energy, the excitation energy and the neutron binding energy, because the neutron decays are forbidden and gamma decays are much smaller than the proton emission even below the Coulomb barrier. The PNEM is shown schematically in Figure 1A. The analysis of the mass-number distribution of ${ }^{100-x} \mathrm{Nb}$ shows the same GR1 and GR2 as in case of the NEM analysis. The missing $\mathrm{Zr}$ isotopes produced by a proton emission in experimental data are predicted by PNEM for both ${ }^{\text {nat }}$ Mo (Ejiri et al., 2013) and ${ }^{100}$ Mo (Hashim et al., 2018). The OMC strength distribution shown in Figure 1A reproduces the observed radioactive isotope (RI) mass distribution.

Since then, various calculations have been made for understanding the formation of the GR populated by muon capture reactions in 98, $<A<209$ (Hashim et al., 2020). These calculations have been compared with previous experimental works by OMC on the nuclei reported in references (Measday, 2001; Measday et al., 2007b; Measday et al., 2007a). The parameters of $E_{G 1}$ and $E_{G 2}$ as a function of $A$ are given as $E_{G 1}=30 A^{-1 / 5}$ and $E_{G 2}=$ $75 A^{-1 / 5}$ for OMC on ${ }^{\text {nat }} \mathrm{Mo},{ }^{100} \mathrm{Mo},{ }^{107} \mathrm{Pd},{ }^{108} \mathrm{Pd},{ }^{127} \mathrm{I}$ and ${ }^{209} \mathrm{Bi}$. They 
are obtained from a comparison of PNEM calculations with experimental data. The observed mass distributions agree with the predictions as shown in Figure 2.

\section{IMPLICATIONS FOR DOUBLE BETA DECAYS}

The experimental OMC rate for ${ }^{100} \mathrm{Mo}$ as a function of the excitation energy is compared with the theoretical calculation by using protonneutron quasi-particle random-phase approximation (pn-QRPA) (Jokiniemi et al., 2019). The pn-QRPA well reproduces the GR at around $10-14 \mathrm{MeV}$ with mainly low multipoles of $J^{\pi}=0^{ \pm}, 1^{ \pm}$and $2^{ \pm}$. The observed OMC rate is the same order of magnitude as the empirical value suggested by Primakoff (Suzuki et al., 1987) but is lower by a factor 5 than the pn-QRPA (Jokiniemi et al., 2019; Jokiniemi and Suhonen, 2019). This suggests a quenching coefficient for the axial-vector weak coupling of $g_{A}^{e f f} / g_{A} \approx 0.5$ (Jokiniemi et al., 2019; Jokiniemi and Suhonen, 2019) for $\mu$-NMEs, being consistent with the quenching factors for Gamow-Teller (GT) and spin dipole (SD) NMEs (Ejiri and Suhonen, 2015; Ejiri et al., 2019). Here the quenching is common in the wide momentum region (Ejiri, 2019a; Ejiri, 2019b). The quenching effects are also discussed theoretically (Menéndez et al., 2011; Suhonen, 2017; Ejiri et al., 2019).

It is interesting to note that the OMC results together with the $\left({ }^{3} \mathrm{He}, \mathrm{t}\right) \mathrm{CERs}$ and $\beta^{ \pm}$data suggest severe quenching of experimental axial-vector NMEs of $M_{i}^{-}$and $M_{i}^{+}$for multipoles of $J^{\pi}=1^{+}, 2^{-}, 3^{+}$ (Jokiniemi et al., 2019; Jokiniemi and Suhonen, 2019). The common quenching coefficients of $k_{N M}=g_{\mathrm{A}}{ }^{\text {eff }} / g_{A}$ for them are around 0.4-0.6 with respect to the pn-QRPA. In this case, the axial-vector DBD NMEs are quenched by the coefficient $k_{N M}^{2}$ and the axial-vector antineutrino NMEs by $k_{N M}$. On the other hand, the recent pn-QRPA calculations for OMC rates show no quenching (Šimkovic et al., 2020) or only weak quenching (Ciccarelli et al., 2020). The different quenching coefficients are partly due to the different model space and the different muon-wave functions used in their QRPA calculations (Jokiniemi et al., 2019; Jokiniemi and Suhonen, 2019; Ciccarelli et al., 2020; Šimkovic et al., 2020). Further studies are needed to see how pn-QRPA calculations reproduce both the relative and absolute OMC rates.

\section{REMARKS AND PERSPECTIVES}

The relative strength distribution of OMC on Mo isotopes show the $\mu$-GR around $E \approx 12 \mathrm{MeV}$ consistent with the pn-QRPA calculation (Jokiniemi et al., 2019). However, the absolute strength derived from the lifetime is much smaller than the

\section{REFERENCES}

Ciccarelli, M., Minato, F., and Naito, T. (2020). Theoretical Study of Nb Isotope Productions by Muon Capture Reaction on Mo100. Phys. Rev. C 102, 034306. doi:10.1103/PhysRevC.102.034306 model value, suggesting a similar quenching of the $M_{i}^{+}$NMEs as the $M_{i}^{-}$NMEs derived from light-ion CERs. Another pnQRPA (Šimkovic et al., 2020) shows no severe quenching. The quenching for $\mu$ NMEs remains to be studied.

The extensive experimental programs on OMC for other nuclei of DBD and supernova anti-neutrino interests are under progress at RCNP and Paul Scherrer Institute (PSI), Switzerland, by the join group of Joint Institute for Nuclear Research (JINR), Dubna, RCNP, Osaka and Universiti Teknologi Malaysia (UTM) (Hashim and Ejiri, 2019). The pnQRPA theoretical calculation is also in progress at Jyvaskyla (Jokiniemi et al., 2019).

Finally, it is remarked that muon capture isotope production (MuCIP) is used for producing efficiently nuclear isotopes with the atomic number $Z-1$, less by one than the atomic number $Z$ of the target isotope. By using ${ }_{Z}^{A} \mathrm{X}$ target isotopes, isotopes of ${ }_{Z-1}^{A-1} \mathrm{X}$ are preferentially produced, and several isotopes with $A, A-2, A-3, A-4$ are also produced (Ejiri et al., 2013; Zinatulina et al., 2019). There is potential for using them for basic and applied science. One RI used for medical checks is the ${ }^{99} \mathrm{Mo}$, which is well produced by OMC on ${ }^{100} \mathrm{Mo}$, as studied at RCNP (Ejiri et al., 2013). MuCIP is complementary to photon capture reactions where isotopes of ${ }_{Z}^{A-1} \mathrm{X}$ are well produced (Ejiri et al., 2011; Szpunar et al., 2013). Note that ppb-level nuclei (impurities) are identified by measuring gamma rays from OMC, which are characteristic of the nuclei, as explained in (Ejiri et al., 2013).

\section{AUTHOR CONTRIBUTIONS}

IH and HE are equally accounted for the content of the work.

\section{FUNDING}

This work was financially supported by the Universiti Teknologi Malaysia research grants (Q.J130000.3026.01M14 and R.J130000.7854.5F227) and the Ministry of Higher Education Malaysia Fundamental Research Grant Scheme (FRGS/1/2019/ STG02/UTM/02/6).

\section{ACKNOWLEDGMENTS}

We are grateful to RCNP, UTM, and JINR colleagues for the collaboration and valuable discussions.

Ejiri, H., and de Voight, M. J. A. (1989). Gamma Ray and Electron Spectroscopy in Nuclear Physics, Oxford: Oxford University Press.

Ejiri, H., and Suhonen, J. (2015). "GT Neutrino-Nuclear Responses for Double Beta Decays and Astro Neutrinos. J. Phys. G: Nucl. Part. Phys., 42, 055201. doi:10. 1088/0954-3899/42/5/055201 
Ejiri, H., Shima, T., Miyamoto, S., Horikawa, K., Kitagawa, Y., Asano, Y., et al. (2011). Resonant Photonuclear Reactions for Isotope Transmutation. J. Phys. Soc. Jpn. 80, 094202. doi:10.1143/JPSJ.80.094202

Ejiri, H., H. Hashim, I., Hino, Y., Kuno, Y., Matsumoto, Y., Ninomiya, K., et al. (2013). Nuclear $\gamma$ Rays from Stopped Muon Capture Reactions for Nuclear Isotope Detection. J. Phys. Soc. Jpn. 82, 044202. doi:10.7566/JPSJ.82.044202

Ejiri, H., Suhonen, J., and Zuber, K. (2019). Neutrino-nuclear Responses for AstroNeutrinos, Single Beta Decays and Double Beta Decays. Phys. Rep. 797, 1-102. doi:10.1016/j.physrep.2018.12.001

Ejiri, H. (2000). Nuclear Spin Isospin Responses for Low-Energy Neutrinos. Phys. Rep. 338, 265-351. doi:10.1016/S0370-1573(00)00044-2

Ejiri, H. (2019a). Axial-vector Weak Coupling at Medium Momentum for Astro Neutrinos and Double Beta Decays. J. Phys. G: Nucl. Part. Phys. 46, 125202. doi:10.1088/1361-6471/ab4dcb

Ejiri, H. (2019b). Nuclear Matrix Elements for $\beta$ and $\beta \beta$ Decays and Quenching of the Weak Coupling $g_{A}$ in QRPA. Front. Phys. 7, 30. doi:10.3389/fphy.2019. 00030

Ejiri, H. (2020). Neutrino-mass Sensitivity and Nuclear Matrix Element for Neutrinoless Double Beta Decay. Universe 6, 225. doi:10.3390/universe6120225

Evans, H. J. (1973). Gamma-rays Following Muon Capture. Nucl. Phys. A 207, 379-400. doi:10.1016/0375-9474(73)90354-0

Frekers, D., and Alanssari, M. (2018). Charge-exchange Reactions and the Quest for Resolution. Eur. Phys. J. A. 54, 177. doi:10.1140/epja/i2018-12612-5

Hashim, I. H., and Ejiri, H. (2019). New Research Project with Muon Beams for Neutrino Nuclear Responses and Nuclear Isotopes Production. AAPPS Bull. 29, 21-26. doi:10.22661/AAPPSBL.2019.29.3.21

Hashim, I. H., Ejiri, H., Othman, F., Saroni, S. S., Amelia, W. N., Hamzah, S. A., et al. (2017). Statistical Neutron Emission Model for Neutrino Nuclear Response. EPJ Web Conf. 156, 00005. doi:10.1051/epjconf/201715600005

Hashim, I. H., Ejiri, H., Shima, T., Takahisa, K., Sato, A., Kuno, Y., et al. (2018). Muon Capture Reaction on Mo100 to Study the Nuclear Response for Double$\beta$ Decay and Neutrinos of Astrophysics Origin. Phys. Rev. C 97, 014617. doi:10. 1103/PhysRevC.97.014617

Hashim, I. H., Ejiri, H., Othman, F., Ibrahim, F., Soberi, F., Ghani, N., et al. (2020). Nuclear Isotope Production by Ordinary Muon Capture Reaction. Nucl. Instr. Methods Phys. Res. Section A: Acc. Spectrometers, Detectors Associated Equipment 963, 163749. doi:10.1016/j.nima.2020.163749

Hashim, I. H. (2015). A Study of Weak Nuclear Response by Nuclear Muon capture. PhD Thesis. Osaka: Osaka University.

Hino, Y., Kuno, Y., Sato, A., Sakamoto, H., Matsumoto, Y., Tran, N. H., et al. (2014). “A Highly Intense Dc Muon Source, Music and Muon Clfv Search. Nucl. Phys. B - Proc. Supplements 253-255, 206-207. doi:10.1016/j.nuclphysbps.2014. 09.051

Ibrahim, F. (2018). Excitation Energies of Compound Nucleus Following Ordinary Muon Capture on Medium Heavy Nuclei within Neutron Statistical Model. Thesis. Johor: Universiti Teknologi Malaysia

Jokiniemi, L., and Suhonen, J. (2019). Muon-capture Strength Functions in Intermediate Nuclei of $0 v \beta \beta$ Decays. Phys. Rev. C 100, 014619. doi:10.1103/ PhysRevC.100.014619
Jokiniemi, L., Suhonen, J., Ejiri, H., and Hashim, I. H. (2019). Pinning Down the Strength Function for Ordinary Muon Capture on 100Mo. Phys. Lett. B 794, 143-147. doi:10.1016/j.physletb.2019.05.037

Kortelainen, M., and Suhonen, J. (2004). Nuclear Muon Capture as a Powerful Probe of Double-Beta Decays in Light Nuclei. J. Phys. G: Nucl. Part. Phys. 30, 2003-2018. doi:10.1088/0954-3899/30/12/017

Measday, D. F., Stocki, T. J., Alarcon, R., Cole, P. L., Djalali, C., and Umeres, F. (2007a). Comparison of Muon Capture in Light and in Heavy Nuclei. AIP Conf. Proc. 947, 253-257. doi:10.1063/1.2813812

Measday, D. F., Stocki, T. J., and Tam, H. (2007b). Trays from Muon Capture in I, Au, and Bi. Phys. Rev. C 75, 045501. doi:10.1103/PhysRevC.75.045501

Measday, D. F., Stocki, T. J., Moftah, B. A., and Tam, H. (2007c). $\gamma$ Rays from Muon Capture inAl27and Natural Si. Phys. Rev. C 76, 035504. doi:10.1103/PhysRevC. 76.035504

Measday, D. F. (2001). The Nuclear Physics of Muon Capture. Phys. Rep. 354, 243-409. doi:10.1016/S0370-1573(01)00012-6

Menéndez, J., Gazit, D., and Schwenk, A. (2011). Chiral Two-Body Currents in Nuclei: Gamow-Teller Transitions and Neutrinoless Double-Beta Decay. Phys. Rev. Lett. 107, 062501. doi:10.1103/PhysRevLett.107.062501

Muslim, N. F. H. (2018). Neutron Statistical Model for Muon Capture on Light Nuclei. Thesis. Johor: Universiti Teknologi Malaysia.

Šimkovic, F., Dvornický, R., and Vogel, P. (2020). Muon Capture Rates: Evaluation within the Quasiparticle Random Phase Approximation. Phys. Rev. C 102, 034301. doi:10.1103/PhysRevC.102.034301

Suhonen, J. (2017). Impact of the Quenching of gA on the Sensitivity of $0 v \beta \beta$ Experiments. Phys. Rev. C 96, 055501. doi:10.1103/PhysRevC.96.055501

Suzuki, T., Measday, D. F., and Roalsvig, J. P. (1987). Total Nuclear Capture Rates for Negative Muons. Phys. Rev. C 35, 2212-2224. doi:10.1103/PhysRevC.35. 2212

Szpunar, B., Rangacharyulu, C., Daté, S., and Ejiri, H. (2013). Estimate of Production of Medical Isotopes by Photo-Neutron Reaction at the Canadian Light Source. Nucl. Instr. Methods Phys. Res. Section A: Acc. Spectrometers, Detectors Associated Equipment 729, 41-50. doi:10.1016/j.nima.2013.06.106

Vergados, J. D., Ejiri, H., and Šimkovic, F. (2012). Theory of Neutrinoless DoubleBeta Decay. Rep. Prog. Phys. 75, 106301. doi:10.1088/0034-4885/75/10/106301

Zinatulina, D., Brudanin, V., Egorov, V., Petitjean, C., Shirchenko, M., Suhonen, J., et al. (2019). Ordinary Muon Capture Studies for the Matrix Elements in $\beta \beta$ Decay. Phys. Rev. C 99, 024327. doi:10.1103/PhysRevC.99.024327

Conflict of Interest: The authors declare that the research was conducted in the absence of any commercial or financial relationships that could be construed as a potential conflict of interest.

Copyright (c) 2021 Hashim and Ejiri. This is an open-access article distributed under the terms of the Creative Commons Attribution License (CC BY). The use, distribution or reproduction in other forums is permitted, provided the original author(s) and the copyright owner(s) are credited and that the original publication in this journal is cited, in accordance with accepted academic practice. No use, distribution or reproduction is permitted which does not comply with these terms. 